\title{
Performed Specimen Collection
}

National Cancer Institute

\section{Source}

National Cancer Institute. Performed Specimen Collection. NCI Thesaurus. Code C93435.

The completed action of gathering samples that may be used for subsequent analysis. 